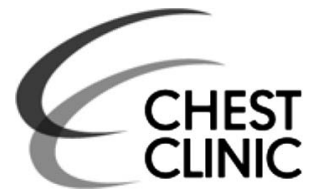

${ }^{1}$ Emergency Department, Derriford Hospital, Plymouth, UK

${ }^{2}$ Academic Department of Military Medicine, Royal Centre for Defence Medicine, Birmingham, UK

\section{Correspondence to} Dr Edward B G Barnard, Emergency Department, Derriford Hospital, Level 6 Plymouth PL6 8DH, UK; barnard@doctors.org.uk

Published Online First 22 January 2013

PULMONARY PUZZLES

\title{
Pulmonary puzzle: a precordial crunch
}

\author{
Edward B G Barnard, ${ }^{1}$ Joanna Rimmer, ${ }^{2}$ Andrew McD Johnston ${ }^{2}$
}

Three patients with unusual additional sounds heard on auscultation of the precordium were admitted to a military hospital in Afghanistan.

Patient 1: A male in his 20s was shot in his left flank. Injuries included a left haemothorax and visceral injuries. An intercostal drain (ICD) was inserted. After surgery, he was admitted to the intensive care unit (ICU) where a 'click' was heard on auscultation of the precordium just after the second heart sound, loudest in expiration. A chest radiograph was taken (figure 1).

Patient 2: A male in his 20s was very close to an explosion. He had fragmentation wounds to his chest, abdomen and limbs. Initial CT scan demonstrated a right-side haemopneumothorax and fragmentation within his pleural space. Intraoperatively, a right-sided ICD was inserted. In the ICU, a 'crunch' was heard over the precordium loudest in expiration and synchronous with the heart sounds. A second CT was performed (figure 2).

Patient 3: A male in his 30s was close to an explosion. He sustained facial fractures and fragmentation injuries. Initial CT demonstrated multiple fractures and soft tissue injuries to the face, but a normal mediastinum, heart and lungs. Following surgery he was transferred to the ICU. Two days later, a systolic 'click' was heard on auscultation of the precordium. Cardiac ultrasound showed no mediastinal or pericardial abnormality. A chest radiograph was taken (figure 3).

\section{QUESTIONS}

1. What eponym is associated with the additional sounds described? What is its significance?

2. Describe the abnormalities in these images.

\section{ANSWERS}

1. A systolic 'crunch' or 'click' that varies with respiration is called Hamman's sign. It is found in Hamman's syndrome, spontaneous pneumomediastinum, but also in patients with pneumothorax.

In 1937, Louis Hamman reported a series of seven patients who had an added sound heard over the precordium. ${ }^{1}$ After examination and radiographs, he proposed that this distinctive sound, described as a 'crunching, crackling, clicking or popping', was indicative of pneumomediastinum with or without concurrent pneumothorax. ${ }^{12}$ Modern imaging has shown that Hamman's sign may occur independently in association with both pneumomediastinum and pneumothorax. ${ }^{3}$ The added sound is thought to be caused when extrapulmonary air is moved between the intrathoracic structures
To cite: Barnard EBG, Rimmer J, McD Johnston $A$. Thorax 2013;68:794-795.

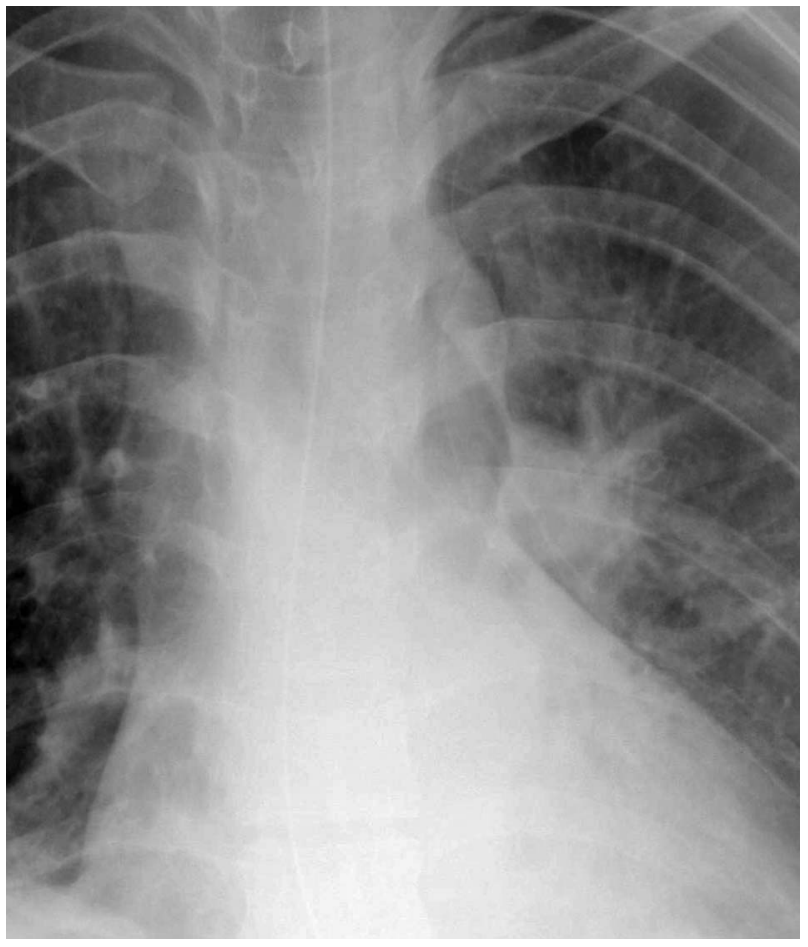

Figure 1 A chest radiograph of patient 1. 


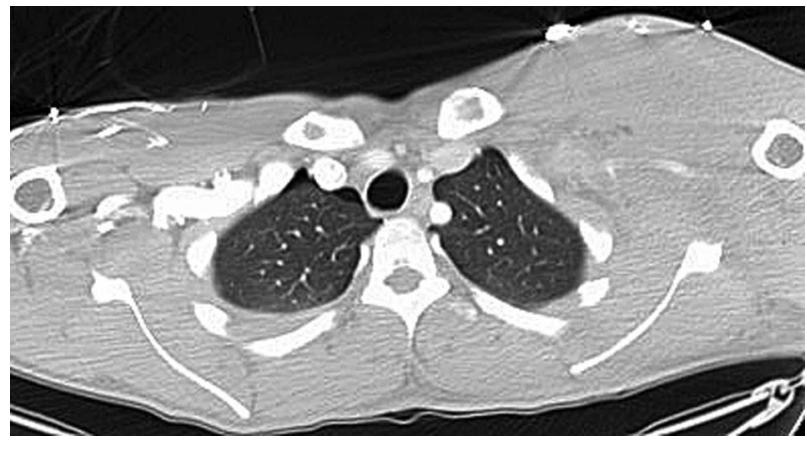

Figure 2 A CT scan of patient 2.

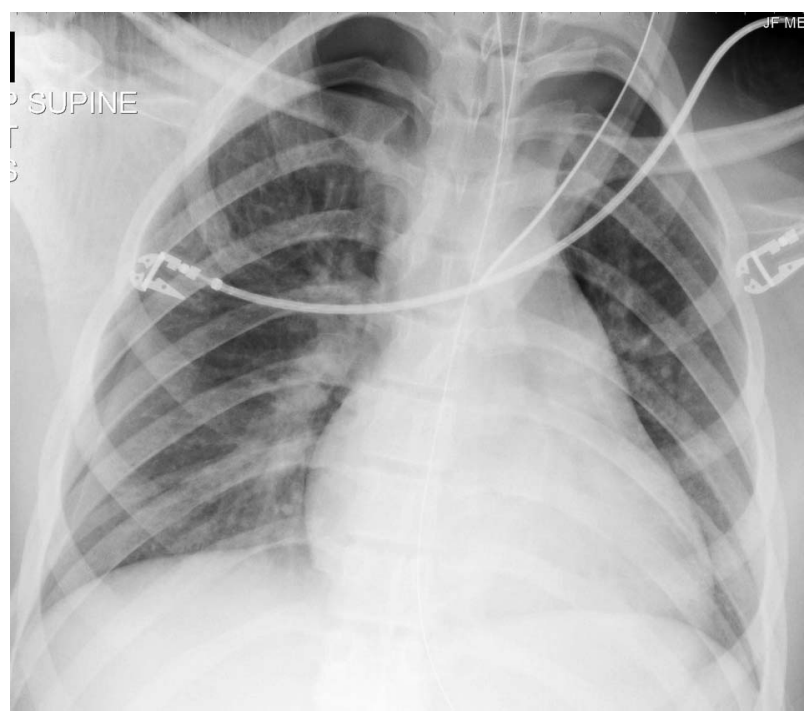

Figure 3 A chest radiograph of patient 3. during the cardiac cycle. ${ }^{3}$ The sound varies with the respiratory cycle, is often positional, usually loudest over the left side of the precordium, and is most pronounced in expiration. $^{1-3}$

2. The first patient's chest radiograph demonstrates a pneumomediastinum, visible as a rounded lucency to the left of the mediastinum. He made a full recovery without further intervention. The second patient's CT shows bilateral anterior pneumothoraces. He made a full recovery with conservative management of his pneumothoraces. The third patient's chest radiograph shows lucencies consistent with bilateral apical pneumothoraces. He required bilateral ICDs for safe aeromedical transfer to another facility.

The cases we describe had experienced pulmonary trauma causing rupture of alveoli and pulmonary interstitial emphysema leading to pneumothorax and/or pneumomediastinum. The misconception that a precordial 'crunch' is found only in pneumomediastinum, continues in medical textbooks. ${ }^{4}$

Hamman's sign is an important clinical indicator of pneumothorax, or pneumomediastinum, which, in patients with lung trauma may require placement of an ICD, particularly if a period of positive pressure ventilation or evacuation by air is contemplated, where there is a risk of expanding gas causing tension pneumothorax.

Information has been removed from these case descriptions to ensure the patients' anonymity.

Contributors All authors contributed equally to the writing of this article.

Competing interests None.

Provenance and review Not commissioned; internally peer reviewed.

\section{REFERENCES}

1 Hamman L. Spontaneous mediastinal emphysema. Bull Johns Hopkins Hosp 1939;64:1-21.

2 Hamman L. A note on the mechanism of spontaneous pneumothorax. Ann Intern Med 1939;13:923-7.

3 Baumann MH, Sahn SA. Hamman's sign revisited. Pneumothorax or pneumomediastinum? Chest 1992;102:1281-2.

4 Marx JA, Hockberger RS, Walls RM. Rosen's Emergency Medicine: concepts and clinical practice. Philadelphia, Pennsylvania, USA: Mosby Elsevier, 2010:1061. 